\title{
TOPSIS with fuzzy belief structure for group belief multiple criteria decision making
}

\author{
Jiang Jiang ${ }^{a, b, *}$, Yu-wang Chen ${ }^{b}$, Ying-wu Chen ${ }^{\text {a }}$, Ke-wei Yang ${ }^{\text {a }}$ \\ ${ }^{a}$ College of Information System and Management, National University of Defense Technology, Changsha 410073, PR China \\ ${ }^{\mathrm{b}}$ Manchester Business School, University of Manchester, Manchester M15 6PB, UK
}

\section{A R T I C L E I N F O}

\section{Keywords:}

Group belief multiple criteria decision

making

TOPSIS

Fuzzy belief Structure (BS) model

Group decision making

Belief distance measure

\begin{abstract}
A B S T R A C T
TOPSIS is one of the major techniques in dealing with multiple criteria decision making (MCDM) problems, and Belief Structure (BS) model and Fuzzy BS model have been used successfully for uncertain MCDM with incompleteness, impreciseness or ignorance. In this paper, the TOPSIS method with Fuzzy BS model is proposed to solve Group Belief MCDM problems. Firstly, the Group Belief MCDM problem is structured as a fuzzy belief decision matrix in which the judgments of each decision maker are described as Fuzzy BS models, and then the Evidential Reasoning approach is used for aggregating the multiple decision makers' judgments. Subsequently, the positive and negative ideal belief solutions are defined with the principle of TOPSIS. In order to measure the separation from the ideal belief solutions, the concept and algorithm of Belief Distance Measure are introduced to compare the difference between Fuzzy BS models. Using the Belief Distance Measure, the relative closeness and ranking index can be calculated for ranking the alternatives. A numerical example is finally given to illustrate the proposed method.
\end{abstract}

(c) 2011 Elsevier Ltd. All rights reserved.

\section{Introduction}

TOPSIS (Technique for Order Performance by Similarity to Ideal Solution) is one of the major techniques in dealing with Multiple Criteria Decision Making (MCDM) problems. It simultaneously considers both the shortest distance from the Positive Ideal Solution (PIS) and the farthest distance from the Negative Ideal Solution (NIS), and preference order is ranked according to their relative closeness combining two distance measures (Hwang \& Yoon, 1981). The technique is helpful for Decision Makers (DMs) to structure the problems, conduct analysis, and rank the alternatives (Shih, Shyur, \& Lee, 2007). Due to its logicality, rationality, and computational simplicity, TOPSIS has been widely applied to the research of evaluation and selection problems and risk analysis problems (Balli \& Korukoglu, 2009; Chamodrakas, Alexopoulou, \& Martakos, 2009; Chen \& Tzeng, 2004; Chen, Lin, \& Huang, 2006; Chu, 2002; Chu \& Lin, 2003; Dagdeviren, Yavuz, \& Kilinc, 2009; Deng, Yeh, \& Willis, 2000; Ertugrul \& Karakasoglu, 2009; Wang \& Elhag, 2006; Wang \& Lee, 2009; Ye \& Li, 2009).

To meet the specific requirements of real-world decision making problems, e.g., uncertain or group decision environments, over past few years many extended TOPSIS methods have also been proposed, such as Fuzzy TOPSIS (Chamodrakas et al., 2009; Chen, 2000; Chen et al., 2006; Chu, 2002; Chu \& Lin, 2003; Dagdeviren

\footnotetext{
* Corresponding author at: College of Information System and Management, National University of Defense Technology, Changsha 410073, PR China. Tel.: +86 13875921240 .

E-mail address: jiangjiangnudt@hotmail.com (J. Jiang).
}

et al., 2009; Jahanshahloo, Lotfi, \& Izadikhah, 2006b; Wang \& Elhag, 2006; Wang \& Lee, 2007; Wang \& Lee, 2009); Fuzzy AHP and TOPSIS (Balli \& Korukoglu, 2009; Ertugrul \& Karakasoglu, 2009); TOPSIS with interval data (Jahanshahloo, Lotfi, \& Izadikhah, 2006a; Ye \& Li, 2009); TOPSIS with grey relation analysis (Chen \& Tzeng, 2004) and Group TOPSIS (Shih et al., 2007; Wang \& Lee, 2007; Ye \& Li, 2009). Particularly, in the stream of Fuzzy TOPSIS, Chen (2000) extended TOPSIS to the fuzzy environment where the rating of each alternative and the weight of each criterion are described by linguistic terms and expressed in triangular fuzzy numbers. The ranking of all alternatives is determined by calculating the distances to both the fuzzy PIS and fuzzy NIS simultaneously. Chu (2002) proposed a fuzzy group TOPSIS model under different subjective attributes in which the membership function is aggregated by interval arithmetic and $\alpha$-cuts of fuzzy numbers and alternatives are ranked by mean of the integral values. It was further applied to the decision problems with criteria assessed in linguistic terms (Chu \& Lin, 2003). Wang and Elhag (2006) introduced a fuzzy TOPSIS method on alpha level sets and presented a nonlinear programming solution procedure. Jahanshahloo et al. (2006b) extended TOPSIS method for decision-making problems with fuzzy data. Wang and Lee (2007) presented a generalized TOPSIS with two operators Up and Lo which satisfy the partial ordering relation on fuzzy numbers. More recently, Fuzzy Analytic Hierarchy Process (FAHP) and TOPSIS methods was proposed by taking subjective judgments of decision makers into consideration by Balli and Korukoglu (2009) and Ertugrul and Karakasoglu (2009). In addition, Fuzzy TOPSIS approach integrating subjective and objective weights (Wang \& Lee, 2009), a class of fuzzy methods based on 
TOPSIS (Chamodrakas et al., 2009), and the AHP and TOPSIS methods under fuzzy environment (Dagdeviren et al., 2009) were also developed for particular decision making problems.

So far, Fuzzy TOPSIS mainly considers the situation where the rating of alternatives is expressed by vague or fuzzy concepts, that is to say, an alternative can only be evaluated by a single linguistic variable, e.g., either Good or Bad, on a certain criterion (Chen, 2000). However, in real-world decision making problems, DMs usually can not express their evaluations by a single linguistic variable due to the incompleteness and ignorance involved in information acquisition process. Therefore, it is more appropriate to express their opinions by a series of linguistic variables with belief degrees, for example, the criterion $C$ of alternative $A$ may be evaluated as (good, 0.7), (average, 0.3)\}, which means that the DM is $70 \%$ sure that criterion $C$ is 'good' and $30 \%$ sure that it is 'average'. This evaluation structure having belief degree on each evaluation grade is called as Belief Structure (BS), which was firstly developed to deal with MCDM problems under uncertainty by Yang and Singh (1994), Yang and Sen (1994), and Yang and Xu (2002), and it has been successfully extended to the area of Fuzzy MCDM (Guo, Yang, Chin, \& Wang, 2009; Yang, Wang, Chin, \& Xu, 2006), Environmental Impact Assessment (Wang, Yang, \& Xu, 2006), New Product Development (Chin, Xu, Yang, \& Lam, 2008; Lam, Chin, Yang, \& Liang, 2007), Quality Function Deployment (Chin, Wang, Yang, \& Poon, 2009), Failure Mode and Effects Analysis (Chin, Wang, Poon, \& Yang, 2009), etc.

This paper is aimed at extending TOPSIS to deal with Group Belief MCDM problem on the basis of the Fuzzy Belief Structure model. The rest of the paper is organized as follows. In Section 2, the Fuzzy BS model and TOPSIS method are introduced as the basis of this research. In Section 3, a novel belief distance measure algorithm, which is used to measure the difference between two Fuzzy BS models, is proposed and several properties are proved. In Section 4, the procedure of the proposed TOPSIS with Fuzzy BS is presented step by step. Finally, a numerical example is given to illustrate the proposed approach in Section 5, and the paper is concluded in Section 6.

\section{A brief introduction to TOPSIS and fuzzy belief structure}

\subsection{TOPSIS method}

TOPSIS is a useful technique in the field of MCDM (Chen and Hwang, 1992). The basic principle of the method is that the selected alternative should have the shortest distance from PIS and the farthest distance from NIS.

Suppose a MCDM problem has $m$ alternatives and $n$ criteria, and the decision matrix is represented as $\left[x_{i j}\right]_{m \times n}$. The procedure of TOPSIS consists of the following steps:

(1) Calculate the normalized decision matrix.

$$
n_{i j}=\frac{x_{i j}}{\sqrt{\sum_{i=1}^{m} x_{i j}^{2}}}, \quad i=1, \ldots, m ; \quad j=1, \ldots, n .
$$

(2) Calculate the weighted normalized decision matrix.

$$
v_{i j}=w_{j} n_{i j}, \quad i=1, \ldots, m ; \quad j=1, \ldots, n .
$$

where $w_{j}$ is the weight of the $j$ th criterion, and $\sum_{j=1}^{n} w_{j}=1$.

(3) Determine the positive ideal and negative ideal solution

$$
\begin{aligned}
& A^{+}=\left\{v_{1}^{+}, \ldots, v_{n}^{+}\right\}=\left\{\max _{i} v_{i j}, j=1, \ldots, n\right\}, \\
& A^{-}=\left\{v_{1}^{-}, \ldots, v_{n}^{-}\right\}=\left\{\min _{i} v_{i j}, \quad j=1, \ldots, n\right\},
\end{aligned}
$$

(4) Calculate the separation measures, using the $n$-dimensional Euclidean distance. The separation of each alternative from PIS is given as

$$
d_{i}^{+}=\left\{\sum_{j=1}^{n}\left(v_{i j}-v_{j}^{+}\right)^{2}\right\}^{\frac{1}{2}}, \quad i=1, \ldots, m .
$$

Similarly, the separation from NIS is given as

$$
d_{i}^{-}=\left\{\sum_{j=1}^{n}\left(v_{i j}-v_{j}^{-}\right)^{2}\right\}^{\frac{1}{2}}, \quad i=1, \ldots, m .
$$

(5) Calculate the relative closeness to the ideal solution.

$$
C_{i}=\frac{d_{i}^{-}}{d_{i}^{+}+d_{i}^{-}}, \quad i=1, \ldots, m .
$$

(6) Rank the preference according to $C_{i}$.

\subsection{Fuzzy belief structure model}

The BS model, which was originally developed by Yang and Sen (1994), Yang and Xu (2002), Yang et al. (2006), is a distributed assessment scheme with belief degrees to represent the performance of an alternative on a criterion.

Suppose a criterion is assessed by a complete set of standards with $N$ evaluation grades, as represented by $H=\left\{H_{1}, H_{2}, \ldots\right.$, $\left.H_{n} \ldots, H_{N}\right\}$, where $H_{n}$ is the $n$th evaluation grade. Without loss of generality, it is assumed that $H_{n}$ is preferred to $H_{n+1}$. A given assessment for criterion $c$ may be mathematically represented as the following distribution:

$S(c)=\left\{\left(H_{n}, \beta_{n}\right), \quad n=1, \ldots, N\right\}$,

where $\beta_{n} \geqslant 0, \sum_{n=1}^{N} \beta_{n} \leqslant 1$, and $\beta_{n}$ is a belief degree. Eq. (1) means the criterion $c$ is assessed to the grade $H_{n}$ with the belief degree $\beta_{n}$. An assessment $S(c)$ is complete if $\sum_{n=1}^{N} \beta_{n}=1$ and incomplete if $\sum_{n=1}^{N} \beta_{n}<1$. A special case is $\sum_{n=1}^{N} \beta_{n}=0$, which represents a complete ignorance on criterion $c$.

In many practical decision situations, the evaluation grades are possibly represented as fuzzy concepts. So it is necessary to extend the BS model to the Fuzzy environments, in which the evaluation grades are represented as fuzzy sets. Suppose a set of fuzzy evaluation grades $\left\{H_{n}, n=1, \ldots, N\right\}$ may be either triangular or trapezoidal fuzzy sets or their combinations. To simplify the discussion without loss of generality, we assume that only two adjacent fuzzy evaluation grades may intersect as shown in Fig. 1.

Since fuzzy evaluation grades and belief degrees are used, the initial $S(c)$ defined in Eq. (1) should be extended to include both fuzzy sets (grades) and belief degrees. The former is used to model fuzziness or vagueness and the later is used to evaluate incompleteness or ignorance. As such, the extended $S(c)$ is referred to as Fuzzy Belief Structure (Yang et al., 2006).

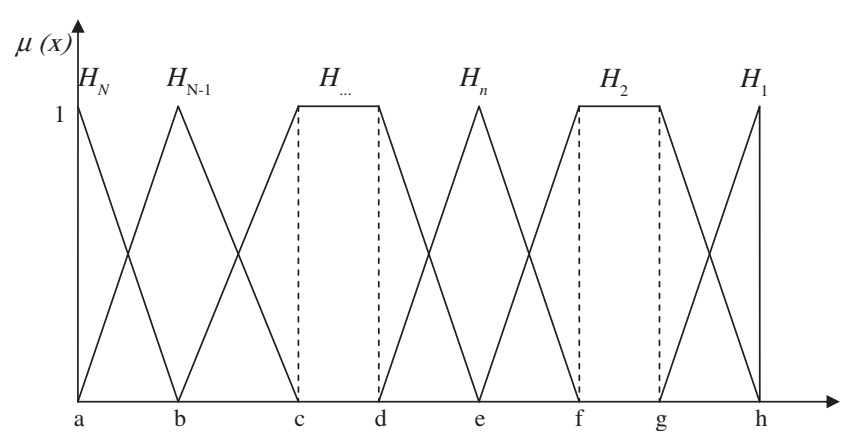

Fig. 1. The mutual relationship between fuzzy evaluation grades. 


\section{Belief distance measure algorithm}

As we know, the distance measure is one of the most important steps in TOPSIS method. In order to extend TOPSIS to deal with Fuzzy BS model, we first define a belief distance measure to quantify the difference between two Fuzzy BS models, and then prove some basic geometrical properties of the belief distance measure in this section.

\subsection{Basic concepts for metric distances}

A metric distance defined on the set $X$ is a mapping function,

$d: X \times X \rightarrow R$

where $R$ is the set of real number. For all $x, y, z \in X$, the distance function is required to satisfy the following conditions:

(1) Non-negativity: $d(x, y) \geqslant 0$;

(2) Identity of indiscernible: $d(x, y)=0$ if and only if $x=y$;

(3) Symmetry: $d(x, y)=d(y, x)$;

(4) Triangle inequality: $d(x, y) \leqslant d(x, z)+d(z, y)$.

In the research field of decision science, Minkowski's $L_{p}$ metric is usually used in many methods, such as goal programming and global criteria method (Abo-Sinna \& Amer, 2005; Lai, Liu, \& Hwang, 1994). The $L_{p}$ metric defines the distance between two points $f$ and $f^{*}$ (the reference point) in $k$-dimensional space as follows:

$d_{p}=\left\{\sum_{i=1}^{n}\left(f_{i}^{*}-f_{i}\right)^{p}\right\}^{1 / p}, \quad p \geqslant 1$.

One physical property of $d_{p}$ measure is well known: when $p$ increases, distance $d_{p}$ decreases, i.e., $d_{1} \geqslant d_{2} \geqslant \cdots \geqslant d_{\infty}$. It means greater emphasis is given to the largest deviation in forming the total distance. More specifically, $p=1$ implies an equal importance (weights) for all these deviations; $p=2$ implies that these deviations are weighted proportionately with the largest deviation having the largest weight. And ultimately $p=\infty$ suggests that the largest deviation completely dominates the distance determination, i.e.,

$d_{\infty}=\max _{i}\left\{\left|f_{i}^{*}-f_{i}\right|\right\}$.

Distance $p=1,2$, and $\infty$ are especially operationally important: $d_{1}$ (the Manhattan distance) and $d_{2}$ (the Euclidean distance) are the longest and the shortest distance in the geometrical sense; $d_{\infty}$ (the Tchebycheff distance) is the shortest distance in the numerical sense.

\subsection{Similarity between fuzzy evaluation grades}

Before defining the belief distance measure, we firstly measure the similarity between fuzzy evaluation grades based on the similarity of fuzzy sets (Chen, 1996; Wei \& Chen, 2009). In Fig. 1, the fuzzy evaluation grades are represented by triangular or trapezoidal fuzzy sets. Correspondingly, the utilities to fuzzy evaluation grades can be also represented by triangular or trapezoidal fuzzy sets, which were called as fuzzy grade utilities (Yang et al., 2006). A fuzzy grade utility should have the same form as its corresponding fuzzy evaluation grade. Without lose of generality, suppose the utilities of each fuzzy evaluation grade are represented using the trapezoidal fuzzy numbers as follows:

$U\left(H_{n}\right)=\left(u_{1}^{n}, u_{2}^{n}, u_{3}^{n}, u_{4}^{n}\right)$

where $u_{k}^{n}(k=1, \ldots, 4) \in[0,1]$. The fuzzy grade utilities corresponding to the fuzzy evaluation grades in Fig. 1 are shown in Fig. 2.

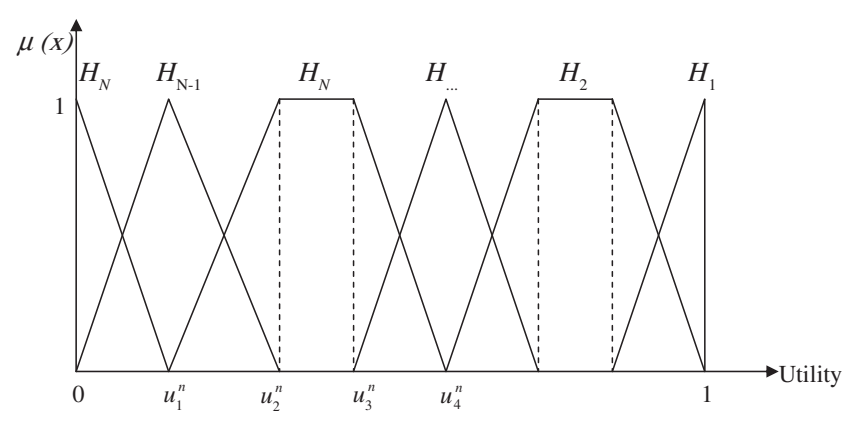

Fig. 2. Fuzzy grade utilities corresponding to fuzzy evaluation grades.

Let $U\left(H_{i}\right)$ and $U\left(H_{j}\right)$ be the fuzzy grade utilities of two fuzzy evaluation grades $H_{i}$ and $H_{j}$, where $U\left(H_{i}\right)=\left(u_{1}^{i}, u_{2}^{i}, u_{3}^{i}, u_{4}^{i}\right)$ and $U\left(H_{j}\right)=\left(u_{1}^{j}, u_{2}^{j}, u_{3}^{j}, u_{4}^{j}\right)$. The similarity between $H_{i}$ and $H_{j}$ is calculated as follows:

$\tilde{s}_{i j}\left(H_{i}, H_{j}\right)=1-\frac{\sum_{k=1}^{4}\left|u_{k}^{i}-u_{k}^{j}\right|}{4}$,

where $0 \leqslant \tilde{s}_{i j} \leqslant 1$, because of $u_{k}^{n}(k=1, \ldots, 4) \in[0,1]$. The similarity describes the difference among the fuzzy evaluation grades. The larger the value of $\tilde{s}_{i j}$, the higher the similarity between the fuzzy evaluation grades $H_{i}$ and $H_{j}$. If a fuzzy evaluation grade $H_{i}$ is triangular fuzzy set, we have $u_{2}^{i}=u_{3}^{i}$ and the similarity calculation is same.

\subsection{Belief distance measure}

According to definition in Eq. (1), a Fuzzy BS model $S$ consists of $N$ fuzzy evaluation grades. To simplify the calculation, we firstly formulate a Fuzzy BS model as a corresponding vector $B=v(S)=\left(\beta_{1}, \beta_{2}, \ldots, \beta_{N}\right)$. In this way, the comparison between two Fuzzy BS models is transformed into the distance measure between two vectors. Suppose there are two Fuzzy BS models $S_{1}$ and $S_{2}$, the corresponding vectors are $B_{1}$ and $B_{2}$. The distance between $S_{1}$ and $S_{2}$ is defined as:

$d_{B S}\left(S_{1}, S_{2}\right)=d_{B S}\left(B_{1}, B_{2}\right)=\left(\frac{1}{2}\left(B_{1}-B_{2}\right) \widetilde{S}\left(B_{1}-B_{2}\right)^{T}\right)^{\frac{1}{2}}$,

where $\widetilde{S}=\left[\tilde{s}_{i j}\right]_{n \times n}$ is a similarity matrix, in which the element $\tilde{s}_{i j}$ is defined in Eq. (3).

This belief distance measure is an effective and simple method to calculate the distance between two Fuzzy BS models. Some important properties are described as follows:

Property 1. The distance between two Fuzzy BS models $S_{1}$ and $S_{2}$ is located between 0 and 1, i.e. $0 \leqslant d_{B S}\left(S_{1}, S_{2}\right) \leqslant 1$.

According to Eq. (4), it is obvious that $d_{B S}\left(S_{1}, S_{2}\right) \geqslant 0$.

Since the quasi-Euclidean distance is normalized by the coefficient $1 / 2$, it can also be guaranteed that $d_{B S}\left(S_{1}, S_{2}\right) \leqslant 1$.

Property 2. Two Fuzzy BS models $S_{1}$ and $S_{2}$ are identical if and only if $d_{B S}\left(S_{1}, S_{2}\right)=0$.

Proof. If $S_{1}$ and $S_{2}$ are identical, $S_{1}=S_{2}$, then $d_{B S}\left(S_{1}, S_{2}\right)=$ $\left(\frac{1}{2} O \widetilde{S} O^{T}\right)^{\frac{1}{2}}=0$.

If $d_{B S}\left(S_{1}, S_{2}\right)=0$, then $\left(B_{1}-B_{2}\right)=0$, which implies $B_{1}=B_{2}$, i.e. $S_{1}=S_{2}$.

Property 3. The distance is symmetrical, i.e. $d_{B S}\left(S_{1}, S_{2}\right)=d_{B S}\left(S_{2}, S_{1}\right)$. 


\section{Proof}

$$
\begin{aligned}
d_{B S}\left(S_{1}, S_{2}\right) & =d_{B S}\left(B_{1}, B_{2}\right)=\left(\frac{1}{2}\left(B_{1}-B_{2}\right) \widetilde{S}\left(B_{1}-B_{2}\right)^{T}\right)^{\frac{1}{2}} \\
& =\left(\frac{1}{2}\left(B_{2}-B_{1}\right) \widetilde{S}\left(B_{2}-B_{1}\right)^{T}\right)^{\frac{1}{2}}=d_{B S}\left(B_{2}, B_{1}\right) \\
& =d_{B S}\left(S_{2}, S_{1}\right) .
\end{aligned}
$$

Property 4. Let $S_{1}, S_{2}$ and $S_{3}$ be three Fuzzy BS models. The triangle inequality is satisfied: $d_{B S}\left(S_{1}, S_{2}\right)+d_{B S}\left(S_{2}, S_{3}\right) \geqslant d_{B S}\left(S_{1}, S_{3}\right)$.

Proof. In order to prove $d_{B S}\left(S_{1}, S_{2}\right)+d_{B S}\left(S_{2}, S_{3}\right) \geqslant d_{B S}\left(S_{1}, S_{3}\right)$, that is to prove

$$
\begin{aligned}
& \sqrt{\frac{1}{2}\left(B_{1}-B_{2}\right) \widetilde{S}\left(B_{1}-B_{2}\right)^{T}}+\sqrt{\frac{1}{2}\left(B_{2}-B_{3}\right) \widetilde{S}\left(B_{2}-B_{3}\right)^{T}} \\
& \geqslant \sqrt{\frac{1}{2}\left(B_{1}-B_{3}\right) \widetilde{S}\left(B_{1}-B_{3}\right)^{T}} .
\end{aligned}
$$

Let $m_{1}=\left(B_{1}-B_{2}\right), m_{2}=\left(B_{2}-B_{3}\right)$, then $\left(B_{1}-B_{3}\right)=m_{1}+m_{2}$, the above inequality is transformed as,

$$
\begin{aligned}
\sqrt{\frac{1}{2} m_{1} \widetilde{S} m_{1}^{T}}+\sqrt{\frac{1}{2} m_{2} \widetilde{S} m_{2}^{T}} & \geqslant \sqrt{\frac{1}{2}\left(m_{1}+m_{2}\right) \widetilde{S}\left(m_{1}+m_{2}\right)^{T}} \\
& =\sqrt{\frac{1}{2}\left(\begin{array}{l}
m_{1} \widetilde{S} m_{1}^{T}+m_{1} \widetilde{S} m_{2}^{T} \\
+m_{2} \widetilde{S} m_{1}^{T}+m_{2} \widetilde{S} m_{2}^{T}
\end{array}\right)} .
\end{aligned}
$$

Calculate the squares of both sides, we further need to prove,

$2 \sqrt{m_{1} \widetilde{S} m_{1}^{T}} \times \sqrt{m_{2} \widetilde{S} m_{2}^{T}} \geqslant m_{1} \widetilde{S} m_{2}^{T}+m_{2} \widetilde{S} m_{1}^{T}$.

Since $\left(m_{1} \widetilde{S} m_{2}^{T}\right)^{T}=m_{2} \widetilde{S}^{T} m_{1}^{T}=m_{2} \widetilde{S} m_{1}^{T}$, we transform the formula above into

$\sqrt{m_{1} \widetilde{S} m_{1}^{T}} \times \sqrt{m_{2} \widetilde{S} m_{2}^{T}} \geqslant m_{1} \widetilde{S} m_{2}^{T}$.

That is to prove

$m_{1} \widetilde{S} m_{1}^{T} m_{2} \widetilde{S} m_{2}^{T}-m_{1} \widetilde{S} m_{2}^{T} m_{1} \widetilde{S} m_{2}^{T} \geqslant 0$.

The left side of the above inequality is equal to,

$$
\begin{aligned}
m_{1} \widetilde{S} m_{1}^{T} m_{2} \widetilde{S} m_{2}^{T}-m_{1} \widetilde{S} m_{2}^{T} m_{1} \widetilde{S} m_{2}^{T}= & m_{1} \widetilde{S}\left(m_{1}^{T} m_{2}-m_{2}^{T} m_{1}\right) \widetilde{S} m_{2}^{T} \\
= & m_{1} \widetilde{S} m_{1}^{T}\left(\left(m_{1}^{T}\right)^{-1} m_{1}^{T} m_{2} m_{2}^{-1}\right. \\
& \left.-\left(m_{1}^{T}\right)^{-1} m_{2}^{T} m_{1} m_{2}^{-1}\right) m_{2} \widetilde{S} m_{2}^{T} \\
= & m_{1} \widetilde{S} m_{1}^{T}\left(1-\left(m_{1}^{T}\right)^{-1} m_{2}^{T} m_{1} m_{2}^{-1}\right) m_{2} \widetilde{S} m_{2}^{T} .
\end{aligned}
$$

Because $S_{1}, S_{2}$ and $S_{3}$ are Fuzzy BS models, the components of vector $B_{1}, B_{2}$ and $B_{3}$ are not more than 1, i.e., $\sum_{n=1}^{N} \beta_{n} \leqslant 1$, according to the definition of Fuzzy BS model.

So, we have $B_{1} \cdot B_{2}^{T} \leqslant 1, B_{2} \cdot B_{3}^{T} \leqslant 1, B_{1} \cdot B_{3}^{T} \leqslant 1$. And similarly, $m_{1} m_{2}^{T} \leqslant 1, m_{1} m_{2}^{T} \leqslant 1$. As a consequence, we have

$\left(m_{1}^{T}\right)^{-1} m_{2}^{T} m_{1} m_{2}^{-1} \leqslant 1, \quad$ and $1-\left(m_{1}^{T}\right)^{-1} m_{2}^{T} m_{1} m_{2}^{-1} \geqslant 0$.

It is also true that,

$m_{1} \widetilde{S} m_{1}^{T} \geqslant 0$ and $m_{2} \widetilde{S} m_{2}^{T} \geqslant 0$.

So, we can finally prove that,

$m_{1} \widetilde{S} m_{1}^{T} m_{2} \widetilde{S} m_{2}^{T}-m_{1} \widetilde{S} m_{2}^{T} m_{1} \widetilde{S} m_{2}^{T}$

$$
=m_{1} \widetilde{S} m_{1}^{T}\left(1-\left(m_{1}^{T}\right)^{-1} m_{2}^{T} m_{1} m_{2}^{-1}\right) m_{2} \widetilde{S} m_{2}^{T} \geqslant 0
$$

i.e.,

$d_{B S}\left(S_{1}, S_{2}\right)+d_{B S}\left(S_{2}, S_{3}\right) \geqslant d_{B S}\left(S_{1}, S_{3}\right)$.

Property 5. Let $S_{1}, S_{2}$ and $S_{3}$ be three Fuzzy BS models. If $S_{1}$ is closer to $S_{3}$ than to $S_{2}$, then $d_{B S}\left(S_{1}, S_{3}\right)<d_{B S}\left(S_{2}, S_{3}\right)$.

We explain this property using an example. Given three BS models:

$S_{1}=\{($ 'good', 0.9), ('average', 0.1), ('bad', 0) $\}$,

$S_{2}=\{($ 'good', 0.5), ('average', 0.5), ('bad', 0) $\}$,

$S_{3}=\{($ 'good', 1), ('average', 0), ('bad', 0) $\}$.

Suppose $\quad u($ 'good' $)=(0.7,1,1,1), \quad u$ ('average' $)=(0.2,0.4,0.6,0.8)$, $u$ ('bad' $)=(0,0,0,0.3)$. The similarity can be calculated using Eq. (3):

$\widetilde{S}=\left[\begin{array}{ccc}1 & 0.575 & 0.150 \\ 0.575 & 1 & 0.575 \\ 0.150 & 0.575 & 1\end{array}\right]$

and the distances are calculated using Eq. (4):

$$
\begin{aligned}
& d_{B S}\left(S_{1}, S_{3}\right)=d_{B S}\left(B_{1}, B_{3}\right)=\left(\frac{1}{2}(-0.1,0.1,0) \widetilde{S}(-0.1,0.1,0)^{T}\right)^{\frac{1}{2}}=0.0652 \\
& d_{B S}\left(S_{2}, S_{3}\right)=d_{B S}\left(B_{2}, B_{3}\right)=\left(\frac{1}{2}(-0.5,0.5,0) \widetilde{S}(-0.5,0.5,0)^{T}\right)^{\frac{1}{2}}=0.3260 .
\end{aligned}
$$

We therefore conclude,

$d_{B S}\left(S_{1}, S_{3}\right)<d_{B S}\left(S_{2}, S_{3}\right)$.

\section{TOPSIS with fuzzy BS model}

Suppose there are $M$ alternatives $A_{i}(i=1, \ldots, M)$ and $K$ DMs $D_{k}(k=1, \ldots, K)$. Each alternative is evaluated by $C_{j}(j=1, \ldots, L)$ criteria. To represent uncertainty, the Fuzzy BS model as defined by Eq. (1) is applied to describe the judgments of DMs. With the above context, the TOPSIS method with Fuzzy BS model for Group Belief MCDM problem is carried out in the following procedures:

Step 1. Formulate the Group Belief MCDM problem by constructing fuzzy belief decision matrix and identifying the weights of criteria and DMs as:

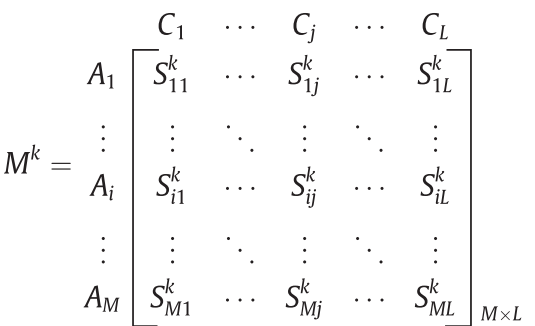

$$
\begin{aligned}
& W^{D}=\left[w_{1}^{D}, \ldots, w_{k}^{D}, \ldots, w_{K}^{D}\right], \\
& W^{C}=\left[w_{1}^{C}, \ldots, w_{j}^{C}, \ldots, w_{L}^{C}\right] \text {, }
\end{aligned}
$$

where $M^{k}$ is the individual fuzzy belief decision matrix for each DM $D_{k}(k=1, \ldots, K) \cdot w_{k}^{D}$ is the weight of DM $D_{k}$, $\sum_{k=1}^{K} w_{k}^{D}=1 . w_{j}^{C}$ is the weight of criterion $C_{j}, \sum_{j=1}^{L} w_{j}^{C}=$ $1 . S_{i j}^{k}=\left\{\left(H_{n}, \beta_{n, k}\right), n=1, \ldots, N\right\}_{i j}$ is a Fuzzy BS model defined in Eq. (1). It means that, in decision maker $D_{k}$ 's opinion, alternative $A_{i}$ is assessed to fuzzy evaluation grade $H_{n}$ with belief degree of $\beta_{n, k}$ regarding criterion $C_{j}$. 
Step 2. Aggregate multiple individual fuzzy belief decision matrix $M^{k}$ into fuzzy belief decision matrix $M$ using the Evidential Reasoning approach (Yang \& Xu, 2002; Yang et al., 2006). The aggregated fuzzy belief decision matrix $M$ can be expressed as:

$M=\left[S_{i j}\right]_{M \times L}=M^{1} \oplus M^{2} \oplus \cdots \oplus M^{K}$,

where its element $S_{i j}$ represents the aggregated rating of alternative $A_{i}$ with respect to criterion $C_{j}$.

In $M=\left[S_{i j}\right]_{M \times L}$ and $M^{k}=\left[S_{i j}^{k}\right]_{M \times L}, S_{i j}$ and $S_{i j}^{k}$ are both Fuzzy BS models, $S_{i j}=\left\{\left(H_{n}, \beta_{n}\right), n=1, \ldots, N\right\}_{i j}$ and $S_{i j}^{k}=\left\{\left(H_{n}, \beta_{n, k}\right), n=\right.$ $1, \ldots, N\}_{i j}$; the analytical relation between $\beta_{n}$ from $\beta_{n, k}$ is expressed as follows (Wang et al., 2006):

$\beta_{n}=\frac{\mu *\left[\prod_{k=1}^{K}\left(w_{k}^{D} \beta_{n, k}+1-w_{k}^{D} \sum_{j=1}^{N} \beta_{j, k}\right)-\prod_{k=1}^{K}\left(1-w_{k}^{D} \sum_{j=1}^{N} \beta_{j, k}\right)\right]}{1-\mu *\left[\prod_{k=1}^{K}\left(1-w_{k}^{D}\right)\right]}$,

$n=1, \ldots, N$

with

$\mu=\left[\sum_{n=1}^{N} \prod_{k=1}^{K}\left(w_{k}^{D} \beta_{n, k}+1-w_{k}^{D} \sum_{j=1}^{N} \beta_{j, k}\right)-(N-1) \prod_{k=1}^{K}\left(1-w_{k}^{D} \sum_{j=1}^{N} \beta_{j, k}\right)\right]^{-1}$,

where $w_{k}^{D}$ is the weight of $k$ th DM in Eq. (6).

Step 3. Normalize Fuzzy BS models if incompleteness is involved. If the Fuzzy BS model is incomplete, i.e. $\sum_{n=1}^{N} \beta_{n}<1$, we define the Center-of-gravity of Fuzzy BS model to deal with this ignorance. Firstly, the degree of ignorance is represented as $\beta_{H}=1-\sum_{n=1}^{N} \beta_{n}$. Then, for the Fuzzy BS model with $N$ Fuzzy evaluation grades, we have $N$ BS peak points, which are defined as:

$S P_{m}(S)=\left\{\left(H_{n}, \beta_{n}^{m}\right), \quad n=1, \ldots, N\right\}, \quad m=1, \ldots, N$

with

$\beta_{n}^{m}= \begin{cases}\beta_{n}+\beta_{H}, & m=n \\ \beta_{n}, & m \neq n\end{cases}$

$S P_{m}(S)$ means a situation when the ignorance degree $\beta_{H}$ of Fuzzy BS model $S$ is assigned entirely to the fuzzy evaluation grade $H_{n}$. Then, the Center-of-gravity of Fuzzy BS model $S$ is defined as:

$$
\begin{aligned}
S C(S) & =\frac{\sum_{m=1}^{N} S P_{m}(S)}{N} \\
& =\left\{\left(H_{n}, \frac{\sum_{m=1}^{N} \beta_{n}^{m}}{N}\right),(n=1, \ldots, N)\right\},
\end{aligned}
$$

when $S$ is incomplete, we use the Center-of-gravity of Fuzzy BS model $S C(S)$ instead of $S$.

Step 4. Determine the Positive Ideal Belief Solutions (PIBS) $A^{+}$and Negative Ideal Belief Solutions (NIBS) $A^{-}$, respectively.

$A^{+}=\left\{S_{1}^{+}, \ldots, S_{j}^{+}, \ldots, S_{L}^{+}\right\}$,

$A^{-}=\left\{S_{1}^{-}, \ldots, S_{j}^{-}, \ldots, S_{L}^{-}\right\}$,

where $S_{j}^{+}$and $S_{j}^{-}$are Fuzzy BS model, and $L$ is the number of criteria.

Step 5. Calculate the separation measure from PIBS $A^{+}$and NIBS $A^{-}$. For each alternative $A_{i}$, the separation measure $D_{i}^{+}$ and $D_{i}^{-}$can be calculated using

$$
\begin{aligned}
D_{i}^{+} & =\sqrt{\sum_{j=1}^{L} w_{j}^{C} d_{B S}\left(S_{i j}, S_{j}^{+}\right)^{2}}, \\
D_{i}^{-} & =\sqrt{\sum_{j=1}^{L} w_{j}^{C} d_{B S}\left(S_{i j}, S_{j}^{-}\right)^{2}}, \quad i=1, \ldots, M,
\end{aligned}
$$

where $w_{j}^{C}$ is the weight of criterion $C_{j}$ in Eq. (7). $d_{B S}\left(S_{i j}, S_{j}^{+}\right)$ (or $d_{B S}\left(S_{i j}, S_{j}^{-}\right)$) are the belief distance measure between the Fuzzy BS model $S_{i j}$ and $S_{j}^{+}$(or $S_{j}^{-}$), which is defined in Eq. (4).

Step 6. Calculate the relative closeness $R_{i}$ for each alternative $A_{i}$ :

$$
R_{i}=\frac{D_{i}^{-}}{D_{i}^{+}+D_{i}^{-}}, \quad i=1, \ldots, M .
$$

Step 7. Rank the preference order according to $R_{i}$. A larger $R_{i}$ indicates a better performance of the alternative.

\section{Numerical example}

In order to illustrate the procedure of TOPSIS method with Fuzzy BS model for Group belief MCDM problem, a numerical example is studied in this section.

Step 1. Structure the Group Belief MCDM problem. Suppose a Group Belief MCDM problem has three alternatives $A_{1}, A_{2}$ and $A_{3}$, four criteria $C_{1}, \ldots, C_{4}$, and three decision makers $D_{1}, D_{2}$ and $D_{3}$. Each decision maker gives his judgment which is expressed as Fuzzy BS model for each alternative on each criterion. Suppose there are four fuzzy evaluation grades $\left\{H_{1}, H_{2}, H_{3}, H_{4}\right\}=\{$ 'excellent', 'good', 'average', 'poor' $\}$. If the decision maker $D_{1}$ state that he is $90 \%$ sure that the alternative $A_{1}$ on the criterion $C_{1}$ is excellent and $10 \%$ sure that it is good, the Fuzzy BS model would be expressed as $\left\{\left(H_{1}, 0.9\right),\left(H_{2}, 0.1\right),\left(H_{3}, 0\right),\left(H_{4}, 0\right)\right\}$, or as $(0.9,0.1,0,0)$ briefly. Similarly, all judgments from decision makers are collected and used to constitute Table 1 . The weights of criteria and DMs are supposed as $W^{C}=[0.3,0.3,0.2,0.2]$ and $W^{D}=[1 / 3,1 / 3,1 / 3]$.

Step 2. Aggregate individual fuzzy belief decision matrix $M^{k}$ into aggregated fuzzy belief decision matrix $M$ using the Evidential Reasoning approach in Eqs. (8) and (9). The results are shown in Table 2.

Step 3. Calculate the Center-of-gravity of Fuzzy BS model for incomplete BS model. From Table 1, we know the Fuzzy BS models $S_{14}^{1}, S_{22}^{1}, S_{24}^{2}, S_{32}^{2}$ and $S_{33}^{3}$ are the incomplete. And correspondingly, $S_{14}, S_{22}, S_{24}, S_{32}$ and $S_{33}$ are incomplete in Table 2. Using the Eqs. (11)-(13), we can update Table 2 and then obtain Table 3.

Step 4. Determine the Positive Ideal Belief Solutions (PIBS) $A^{+}$and Negative Ideal Belief Solutions (NIBS) ideal belief solutions

\begin{tabular}{|c|c|c|c|c|c|}
\hline & & $C_{1}$ & $C_{2}$ & $C_{3}$ & $C_{4}$ \\
\hline \multirow[t]{3}{*}{$A_{1}$} & $D_{1}$ & $0.9,0.1,0,0$ & $0,0.5,0.5,0$ & $0,0.2,0.8,0$ & $0,0.4,0.4,0$ \\
\hline & $D_{2}$ & $0,0.8,0.2,0$ & $1,0,0,0$ & $0,0.4,0.6,0$ & $0,0.7,0.3,0$ \\
\hline & $D_{3}$ & $0,0.6,0.4,0$ & $0,0.9,0.1,0$ & $0,0.2,0.8,0$ & $0,0,0.5,0.5$ \\
\hline \multirow[t]{3}{*}{$A_{2}$} & $D_{1}$ & $0,0.9,0.1,0$ & $0,0.4,0.5,0$ & $1,0,0,0$ & $0.1,0.9,0,0$ \\
\hline & $D_{2}$ & $1,0,0,0$ & $0.3,0.6,0.1,0$ & $0.5,0.5,0,0$ & $0,0.4,0.5,0$ \\
\hline & $D_{3}$ & $0,0.6,0.4,0$ & $0,0.8,0.2,0$ & $1,0,0,0$ & $0.6,0.4,0,0$ \\
\hline \multirow[t]{3}{*}{$A_{3}$} & $D_{1}$ & $0,0.4,0.6,0$ & $0,0.8,0.2,0$ & $0,0.5,0.5,0$ & $0,0,0.8,0.2$ \\
\hline & $D_{2}$ & $0,0.3,0.7,0$ & $0,0.4,0.5,0$ & $0,0,0.4,0.6$ & $0,0.3,0.7,0$ \\
\hline & $D_{3}$ & $0,0.7,0.3,0$ & $0,0,0,1$ & $0,0,0.5,0.4$ & $0,0.2,0.8,0$ \\
\hline
\end{tabular}
$A^{-}$. According to the definition of Fuzzy BS model, the PIBS and NIBS are:

Table 1

BS Judgments from decision makers. 
Table 2

Aggregated fuzzy belief decision matrix.

\begin{tabular}{lllll}
\hline & $C_{1}$ & $C_{2}$ & $C_{3}$ & $C_{4}$ \\
\hline \multirow{2}{*}{$A_{1}$} & $0.2677,0.5419$, & $0.3077,0.5000$, & $0,0.2260$, & $0,0.3641$, \\
& $0.1904,0$ & $0.1923,0$ & $0.7740,0$ & $0.4274,0.1529$ \\
$A_{2}$ & $0.3040,0.5380$, & $0.0836,0.6502$, & $0.8788,0.1212$, & $0.2119,0.6221$, \\
& $0.1581,0$ & $0.2396,0$ & 0,0 & $0.1383,0$ \\
$A_{3}$ & $0,0.4587$, & $0,0.4230$, & $0,0.1469$, & $0,0.1318$, \\
& $0.5413,0$ & $0.2296,0.3172$ & $0.5035,0.3217$ & $0.8185,0.0497$ \\
\hline
\end{tabular}

Table 3

Improved fuzzy belief decision matrix.

\begin{tabular}{lllll}
\hline & $C_{1}$ & $C_{2}$ & $C_{3}$ & $C_{4}$ \\
\hline \multirow{2}{*}{$A_{1}$} & $0.2677,0.5419$, & $0.3077,0.5000$, & $0.0000,0.2260$, & $0.0139,0.3780$, \\
& $0.1904,0.0000$ & $0.1923,0.0000$ & $0.7740,0.0000$ & $0.4413,0.1668$ \\
\multirow{2}{*}{$A_{2}$} & $0.3040,0.5380$, & $0.0902,0.6568$, & $0.8788,0.1212$, & $0.2188,0.6290$ \\
& $0.1581,0.0000$ & $0.2463,0.0066$ & $0.0000,0.0000$ & $0.1452,0.0069$ \\
\multirow{2}{*}{$A_{3}$} & $0.0000,0.4587$, & $0.0076,0.4305$, & $0.0070,0.1539$, & $0.0000,0.1318$, \\
& $0.5413,0.0000$ & $0.2372,0.3247$ & $0.5105,0.3287$ & $0.8185,0.0497$ \\
\hline
\end{tabular}

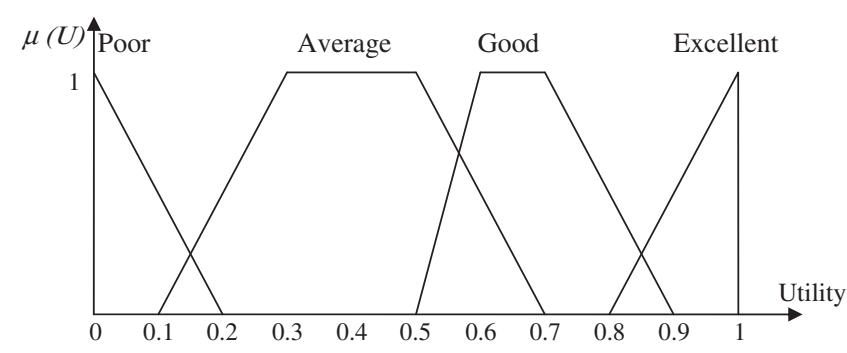

Fig. 3. Fuzzy grade utilities.

Table 4

Belief distances and separation measures.

\begin{tabular}{lllllllll}
\hline & & $C_{1}$ & $C_{2}$ & $C_{3}$ & $C_{4}$ & $D_{i}^{+} / D_{i}^{-}$ & $R_{i}$ & Rank \\
\hline$A_{1}$ & $A^{+}$ & 0.3968 & 0.3768 & 0.6631 & 0.6155 & 0.5035 & 0.5743 & 2 \\
& $A^{-}$ & 0.7416 & 0.7453 & 0.6034 & 0.5341 & 0.6793 & & \\
$A_{2}$ & $A^{+}$ & 0.3743 & 0.4952 & 0.0636 & 0.4174 & 0.3889 & 0.6658 & 1 \\
& $A^{-}$ & 0.7552 & 0.7079 & 0.9151 & 0.7457 & 0.7747 & & \\
\multirow{2}{*}{$A_{3}$} & $A^{+}$ & 0.5963 & 0.6282 & 0.7090 & 0.6951 & 0.6498 & 0.4503 & 3 \\
& $A^{-}$ & 0.6386 & 0.4609 & 0.4060 & 0.5664 & 0.5322 & & \\
\hline
\end{tabular}

$A^{+}=\{1,0,0,0\} \quad$ and $\quad A^{-}=\{0,0,0,1\}$.

Step 5. Calculate the separation measure from the PIBS $A^{+}$and NIBS $A^{-}$.

Suppose the utilities of fuzzy evaluation grades are:

$$
\begin{aligned}
u\left(H_{1}\right) & =u(\text { 'excellent' })=(0.8,1,1,1), \quad u\left(H_{2}\right)=u(\text { 'good' }) \\
& =(0.5,0.6,0.7,0.9),
\end{aligned}
$$

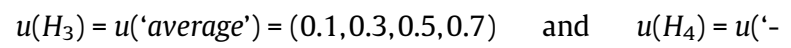
poor' $=(0,0,0,0.2)$, as shown in Fig. 3 .

Then, the similarity matrix can be calculated by Eq. (3):

$$
\widetilde{S}=\left[\begin{array}{llll}
1.000 & 0.725 & 0.450 & 0.100 \\
0.725 & 1.000 & 0.725 & 0.375 \\
0.450 & 0.725 & 1.000 & 0.650 \\
0.100 & 0.375 & 0.650 & 1.000
\end{array}\right]
$$

The distances between decision judgments and ideal solutions are measured by Eq. (4), and the separation measure $D_{i}^{+}$and $D_{i}^{-}$can be calculated using Eq. (15). The results are shown in Table 4.
Step 6. Calculate the relative closeness $R_{i}$ to the ideal solution for each alternative $A_{i}$ using the Eq. (16). The results are also shown in Table 4.

Step 7. Rank the preference order in terms of the values of $R_{i}$. In the numerical example the ranking of three alternatives is $A_{2} \succ A_{1} \succ A_{3}$.

\section{Conclusion}

Uncertainty and group decision prevail in real-world MCDM problems, the former is due to incomplete or non-obtainable information, such as fuzziness, imprecision, vagueness, incompleteness and ignorance; the later is due to the participation of multiple decision makers or experts. Therefore, this paper mainly focuses on the group MCDM problem with uncertainty, which is called Group Belief MCDM problem.

In order to deal with Group Belief MCDM problem, we first study TOPSIS method with Fuzzy Belief Structure model. Fuzzy BS model contains both fuzzy evaluation grades and belief degrees, the former can model fuzziness or vagueness and the later incompleteness or ignorance. And then, the decision making problem is formulated as the fuzzy belief decision matrix with Fuzzy BS models, and Evidential Reasoning approach is used to aggregate multiple decision makers' judgments. To compare the difference between Fuzzy BS models, a novel belief distance measure is defined to measure the separation from ideal belief solutions. Subsequently, the proposed TOPSIS method with Fuzzy BS model is introduced step by step, and is finally illustrated by a numerical example. It is worth noting that the belief distance measure can be not only used for the comparison of BS models in MCDM, but also extended to alternative optimization in the future research.

\section{Acknowledgments}

The author is indebted to the anonymous reviewers for their accurate comments and suggestions that helped improve the work presented in this paper.

\section{References}

Abo-Sinna, M. A., \& Amer, A. H. (2005). Extensions of TOPSIS for multi-objective large-scale nonlinear programming problems. Applied Mathematics and Computation, 162(1), 243-256.

Balli, S., \& Korukoglu, S. (2009). Operating system selection using fuzzy AHP and TOPSIS methods. Mathematical and Computational Applications, 14(2), 119-130. Chamodrakas, I., Alexopoulou, N., \& Martakos, D. (2009). Customer evaluation for order acceptance using a novel class of fuzzy methods based on TOPSIS. Expert Systems with Applications, 36(4), 7409-7415.

Chen, S. M. (1996). New methods for subjective mental workload assessment and fuzzy risk analysis. Cybernetics and Systems, 27(5), 449-472.

Chen, C. T. (2000). Extensions of the TOPSIS for group decision-making under fuzzy environment. Fuzzy Sets and Systems, 114(1), 1-9.

Chen, C. T., Lin, C. T., \& Huang, S. F. (2006). A fuzzy approach for supplier evaluation and selection in supply chain management. International Journal of Production Economics, 102(2), 289-301.

Chen, M. F., \& Tzeng, G. H. (2004). Combining grey relation and TOPSIS concepts for selecting an expatriate host country. Mathematical and Computer Modelling, 40(13), 1473-1490.

Chin, K. S., Wang, Y. M., Poon, G. K. K., \& Yang, J. B. (2009). Failure mode and effects analysis using a group-based evidential reasoning approach. Computers and Operations Research, 36(6), 1768-1779.

Chin, K. S., Wang, Y. M., Yang, J. B., \& Poon, K. K. G. (2009). An evidential reasoning based approach for quality function deployment under uncertainty. Expert Systems with Applications, 36(3), 5684-5694.

Chin, K. S., Xu, D. L., Yang, J. B., \& Lam, J. P. K. (2008). Group-based ER-AHP system for product project screening. Expert Systems with Applications, 35(4), 1909-1929.

Chu, T. C. (2002). Facility location selection using fuzzy TOPSIS under group decisions. International Journal of Uncertainty Fuzziness and Knowledge-Based Systems, 10(6), 687-701.

Chu, T. C., \& Lin, Y. C. (2003). A fuzzy TOPSIS method for robot selection. International Journal of Advanced Manufacturing Technology, 21(4), 284-290.

Dagdeviren, M., Yavuz, S., \& Kilinc, N. (2009). Weapon selection using the AHP and TOPSIS methods under fuzzy environment. Expert Systems with Applications, 36(4), 8143-8151. 
Deng, H., Yeh, C. H., \& Willis, R. J. (2000). Inter-company comparison using modified TOPSIS with objective weights. Computers and Operations Research, 27(10), 963-973.

Ertugrul, I., \& Karakasoglu, N. (2009). Performance evaluation of Turkish cement firms with fuzzy analytic hierarchy process and TOPSIS methods. Expert Systems with Applications, 36(1), 702-715.

Guo, M., Yang, J. B., Chin, K. S., \& Wang, H. W. (2009). Evidential reasoning approach for multi-attribute decision analysis under both fuzzy and interval uncertainty. IEEE Transactions on Fuzzy Systems, 17(3), 683-697.

Hwang, C. L., \& Yoon, K. (1981). Multiple attribute decision making. Berlin: SpringerVerlag.

Jahanshahloo, G. R., Lotfi, F. H., \& Izadikhah, M. (2006a). An algorithmic method to extend TOPSIS for decision-making problems with interval data. Applied Mathematics and Computation, 175(2), 1375-1384.

Jahanshahloo, G. R., Lotfi, F. H., \& Izadikhah, M. (2006b). Extension of the TOPSIS method for decision-making problems with fuzzy data. Applied Mathematics and Computation, 181(2), 1544-1551.

Lai, Y. J., Liu, T. Y., \& Hwang, C. L. (1994). TOPSIS for MODM. European Journal of Operational Research, 76(3), 486-500.

Lam, P. K., Chin, K. S., Yang, J. B., \& Liang, W. (2007). Self-assessment of conflict management in client-supplier collaborative new product development. Industrial Management and Data Systems, 107(5), 688-714.

Shih, H. S., Shyur, H. J., \& Lee, E. S. (2007). An extension of TOPSIS for group decision making. Mathematical and Computer Modelling, 45(7-8), 801-813.

Wang, Y. M., \& Elhag, T. M. S. (2006). Fuzzy TOPSIS method based on alpha level sets with an application to bridge risk assessment. Expert Systems with Applications, 31(2), 309-319.
Wang, Y. J., \& Lee, H. S. (2007). Generalizing TOPSIS for fuzzy multiple-criteria group decision-making. Computers and Mathematics with Applications, 53, 1762-1772.

Wang, T. C., \& Lee, H. D. (2009). Developing a fuzzy TOPSIS approach based on subjective weights and objective weights. Expert Systems with Applications, 36(5), 8980-8985.

Wang, Y. M., Yang, J. B., \& Xu, D. L. (2006). Environmental Impact Assessment Using the Evidential Reasoning Approach. European Journal of Operational Research, 174(3), 1885-1913.

Wei, S. H., \& Chen, S. M. (2009). A new approach for fuzzy risk analysis based on similarity measures of generalized fuzzy numbers. Expert Systems with Application, 36(1), 589-598.

Yang, J. B., \& Sen, P. (1994). A general multi-level evaluation process for hybrid MADM with uncertainty. IEEE Transactions on Systems, Man, and Cybernetics Part A: Systems and Humans, 24(10), 1458-1473.

Yang, J. B., \& Singh, M. G. (1994). An evidential reasoning approach for multiple attribute decision making with uncertainty. IEEE Transactions on Systems, Man, and Cybernetics - Part A: Systems and Humans, 24(1), 1-18.

Yang, J. B., Wang, Y. M., Chin, K. S., \& Xu, D. L. (2006). The evidential reasoning approach for MCDA under both probabilistic and fuzzy uncertainties. European Journal of Operational Research, 171(1), 309-343.

Yang, J. B., \& Xu, D. L. (2002). On the evidential reasoning algorithm for multiattribute decision analysis under uncertainty. IEEE Transactions on Systems, Man, and Cybernetics - Part A: Systems and Humans, 32(3), 289-304.

Ye, F., \& Li, Y. N. (2009). Group multi-attribute decision model to partner selection in the formation of virtual enterprise under incomplete information. Expert Systems with Applications, 36(5), 9350-9357. 